\title{
Deep Reinforcement Learning for optimal energy management of multi-energy Smart Grids
}

\author{
Dhekra Bousnina, Gilles Guerassimoff
}

Center for Applied Mathematics, MINES ParisTech, PSL Research University, France

LOD 2021. October $7^{\text {th }} 2021$

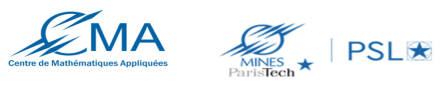

A DEM E

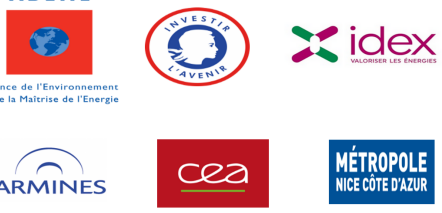




\section{Context of the problem}

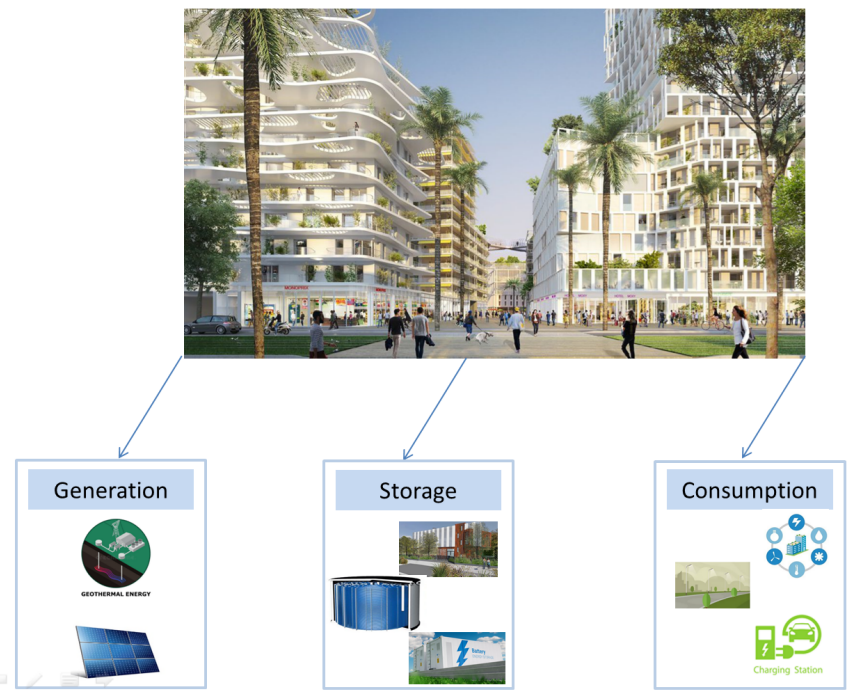

Figure: The Meridia Smart Energy eco-district - Energy systems 


\section{Optimization Objectives}

- Reduce energy consumption, load peaks and energy bills

- Maximize self-consumption and energy self-sufficiency

- Minimize GHG emissions related to energy consumption and mobility

- Value flexibility potentials of the Smart Grid (ancillary services) 


\section{Optimization Objectives}

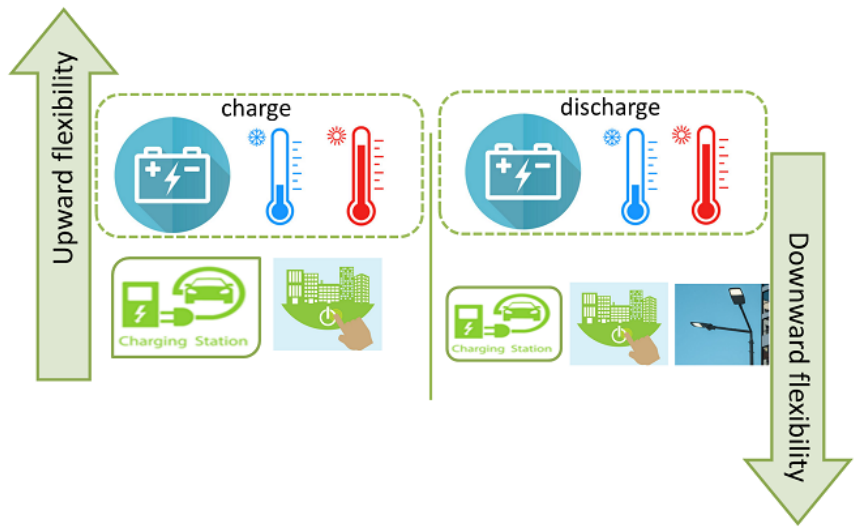

Figure: Flexibility potentials in the MSE Multi-energy Smart Grid 


\section{Optimization objectives}

$15 \mathrm{~min}$

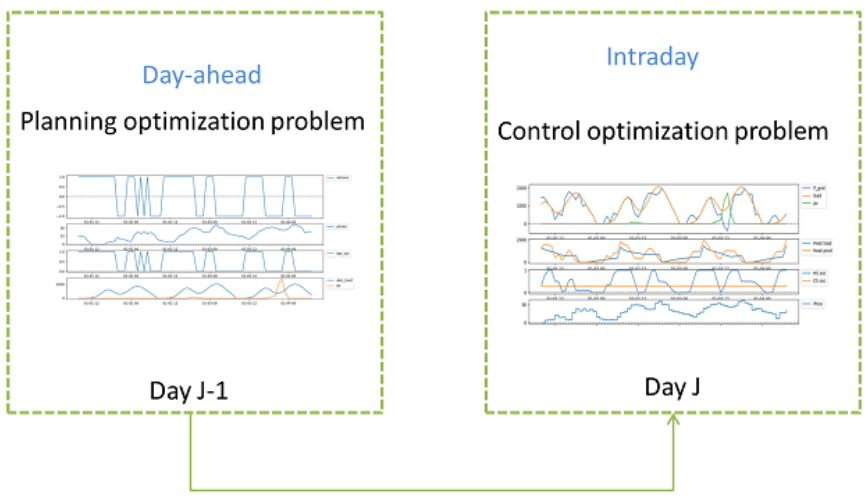

Figure: Optimal planning and optimal control of the energy systems 


\section{This work}

- A wide range of methods: Exact (LP, MILP), predictive (MPC), meta-heuristics (genetic algorithms, PSO)

- Time-consuming, not suitable for on-line solutions, large-scale databases

- Rely on accurate models and predictors, appropriate solvers 


\section{This work}

- A wide range of methods: Exact (LP, MILP), predictive (MPC), meta-heuristics (genetic algorithms, PSO)

- Time-consuming, not suitable for on-line solutions, large-scale databases

- Rely on accurate models and predictors, appropriate solvers

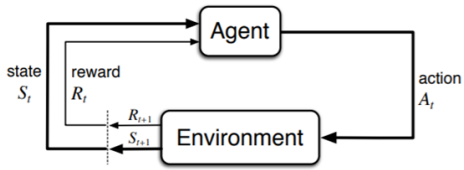

Figure: Reinforcement Learning paradigm (Sutton \& Barto, 2018) 


\section{This work}
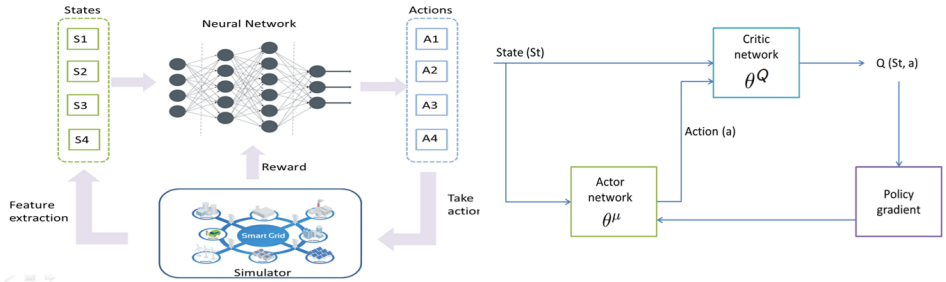

- Renewable energy generation

- Buildings indoor and outdoor

temperatures

- Buildings eletrical and

thermal loads

- State of charge of electrical

and thermal storage systems

State

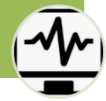

- Actions on geothermal heat pumps ( heat and cold production)

- Actions on PV panels (if curtailable )

- Actions on electrical and thermal storage systems (charge/ discharge)

\section{Actions ( (p) $)_{3}$}

- Cost of energy consumption

- Self-consumption rate

- Income / penalties from participation in ancillary services

\section{Reward}

Figure: Deep Deterministic Policy Gradient 


\section{Input data}
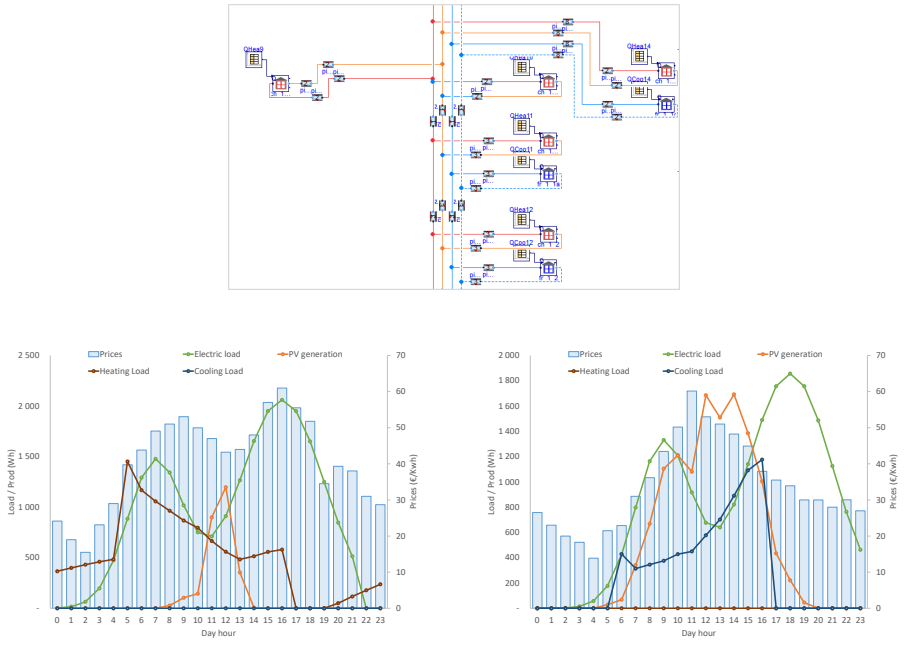

Figure: Example of historical data for a typical winter day/ summer day 


\section{A benchmark solution: MPC}

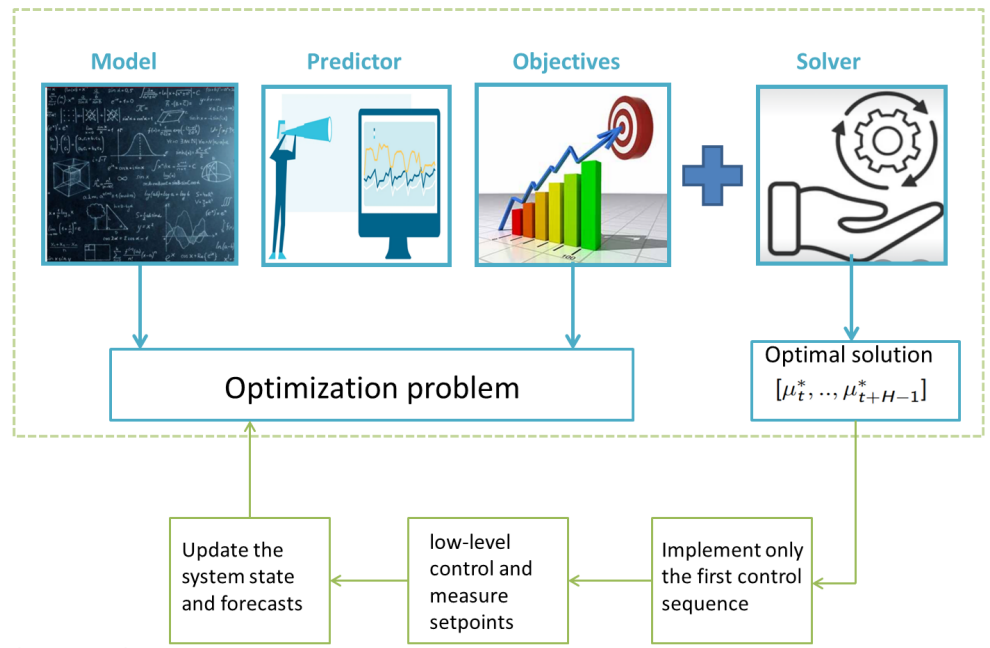

Figure: Model Predictive Control 


\section{Some results}

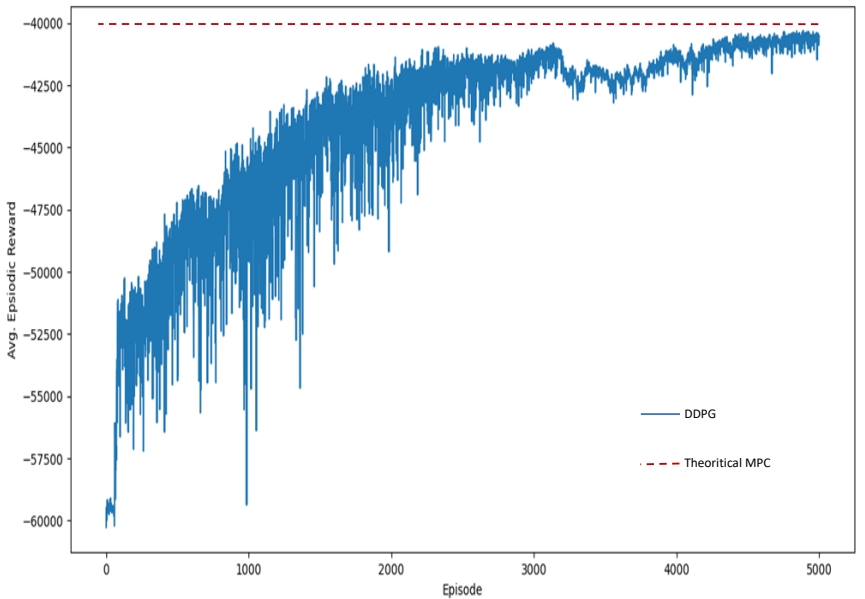

Figure: Learning curve for a 5000 episode training 


\section{Some results}
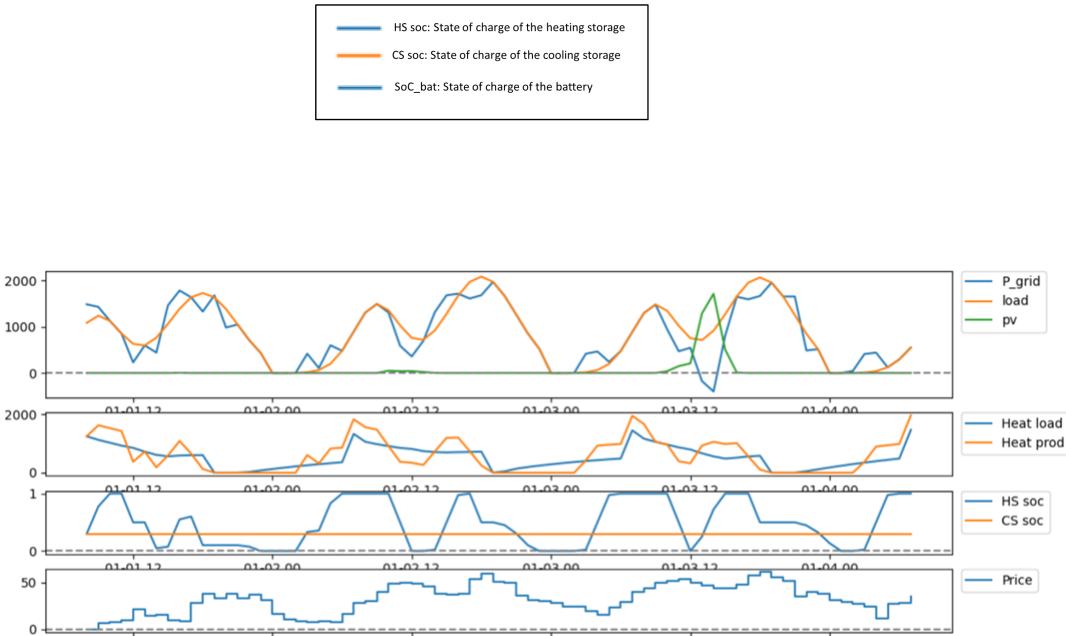

- Heat prod

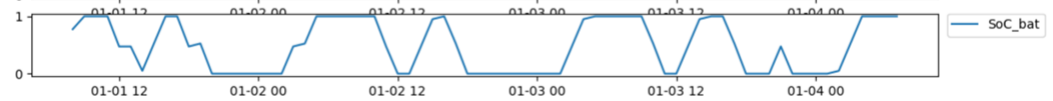




\section{Summary and future work}

- This work proposes a strategy for the optimal operation of a multi-energy Smart Grid

- It uses a DRL approach (Deep Deterministic Policy gradient algorithm)

- Future works include a further comparison of the performance the DRL approach with the benchmark solutions

- The model of the environment will be replaced with the digital twin, then with the district-level real-life Smart Grid 


\section{Thank you for your attention!}

